\title{
Cryo-Electron Microscopy of Outer Membrane Vesicle Production by Vibrio vulnificus
}

\author{
R. Guerrero-Ferreira*, G. M. Williams* and E. R. Wright*, ** \\ * Emory University School of Medicine, Division of Pediatric Infectious Diseases, Children's \\ Healthcare of Atlanta, 2015 Uppergate Drive NE, Atlanta, GA 30322 \\ ** Robert P. Apkarian Integrated Electron Microscopy Core, Emory University, 1521 Dickey \\ Drive, Atlanta, GA 30322
}

Vibrio vulnificus is an opportunistic human pathogen [1]. Its virulence is regulated by cellular appendages (flagellum and pili) as well as other structural components, such as the capsular polysaccharide [2]. Recently, $V$. vulnificus was found to produce outer membrane vesicles (OMVs) that can induce host cell death [3] and may contain a variety of virulence factors [4]. Our goal was to study the formation $V$. vulnificus OMVs using cryo-electron microscopy.

V. vulnificus clinical isolates MO6-24/O and CMCP6 were grown in Luria-Bertani medium supplemented with $2.0 \%(\mathrm{w} / \mathrm{v}) \mathrm{NaCl}$. Cultures were incubated overnight $\left(250 \mathrm{rpm}\right.$ at $\left.28^{\circ} \mathrm{C}\right)$ and flash frozen onto glow-discharged Quantifoil carbon grids in liquid ethane with an FEI Vitrobot. Cryo-EM data was collected with a JEOL 2200FS $200 \mathrm{kV}$, field emission TEM with an incolumn energy filter, on a Gatan 4kx4k high resolution CCD camera.

Cryo-EM data confirms basic cell ultrastructure: the polar placement of the flagellum and chemoreceptor arrays, pili, flagellar core and sheath (Fig. 1). No differences in the pattern of vesicle formation were observed between the two strains. OMVs were frequently observed as 'beads on a string,' with two to twelve vesicles connected. Intra-vesicular material was shared among some OMVs, whereas others appeared closed but still connected to adjacent vesicles (Fig. 2). OMV release occurs as a synchronized and non-polarized process. Vesicle formation was accompanied, in some cases, by the accumulation of particulates within the OMVs. However, no association was observed between the size of emerging vesicles and the amount or size of these particulates. OMVs were either empty or contained variable amounts of material, indicating that the release of an individual OMV is not dependent on the encapsulation of the product they transport (Fig. 3). OMVs were also found to localize around the cell periphery and form concentric rings. This type of vesicle ranged in diameter from 10 to100 nm. They were separated from the cell and from each subsequent OMV ring by a distance of $100 \mathrm{~nm}$ to $200 \mathrm{~nm}$ (Fig. 4).

Future studies will determine whether OMV release is triggered either by environmental conditions or by the accumulation of specific metabolites within the cell. Further structural analyses will characterize OMV contents and identify specific macromolecular complexes on their surface using cryo-electron tomography and sub-tomogram averaging techniques. 
References

[1] G.N. Stelma Jr., et al., Appl. Envrion. Microbiol. 52(1992) 2776-2782.

[2] M.S. Strom, et al., Microbes Infect. 2(2000) 177-88.

[3] Y.R. Kim, et al., Biochem. Biophys. Res. Commun. 399(2010) 607-12.

[4] M.J. Kuehn, et al., Genes Dev. 19(2005) 2645-55.

[5] This research was supported by Emory University, Children's Healthcare of Atlanta, and the Georgia Research Alliance. Cryo-ET data was collected at the Emory University Robert P. Apkarian Integrated Electron Microscopy Core. We would like to thank Dr. Paul Gulig for providing bacterial strains.

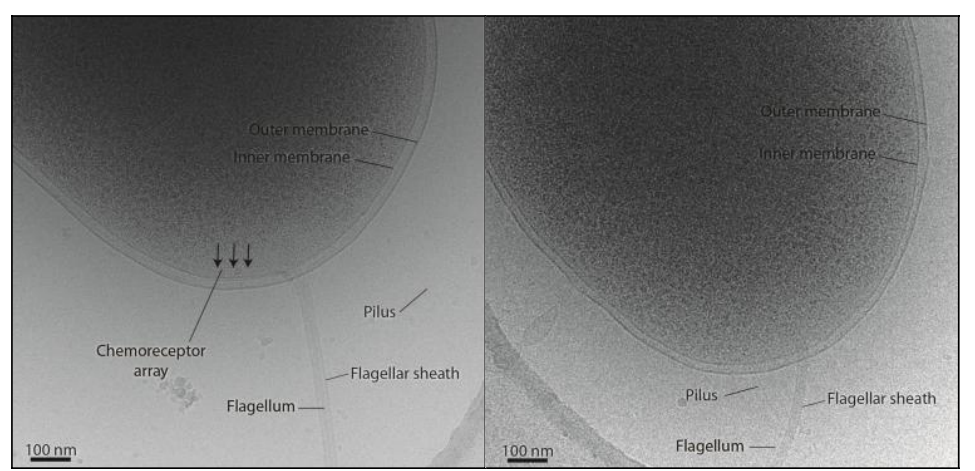

FIG. 1. Cell poles of MO6-24/O (left) and CMCP6 (right). Arrows indicate the chemoreceptor array. Cell membrane and bacterial appendages are also indicated.

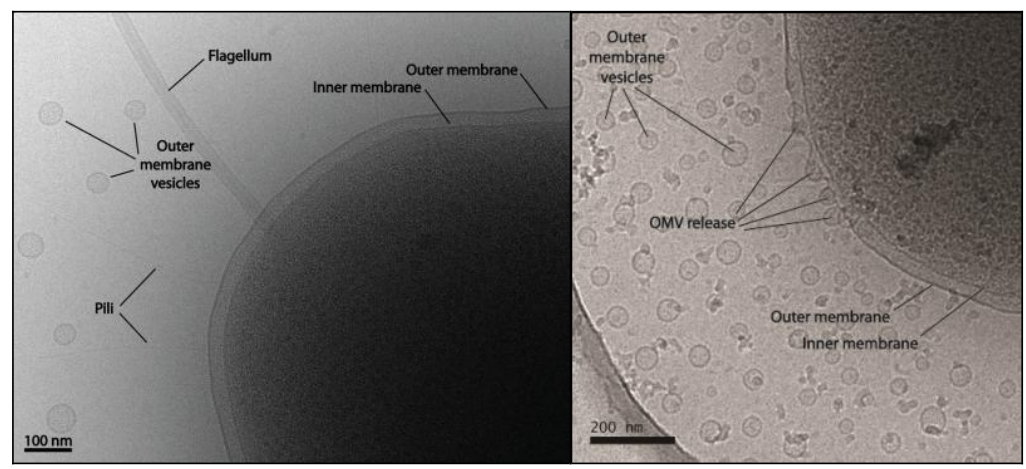

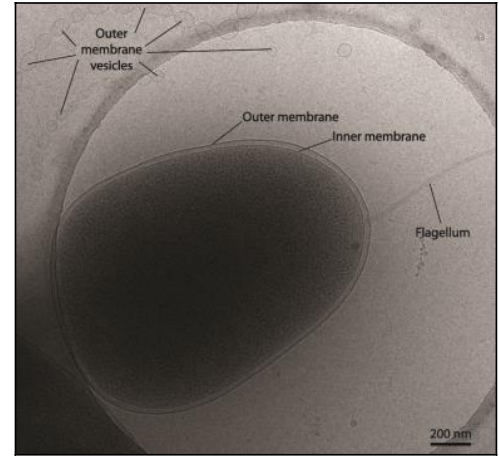

FIG. 2. Cell pole of $V$. vulnificus CMCP6 depicting a filament of OMVs.

FIG. 3. OMVs production in $V$. vulnificus MO6-24/O (left) and CMCP6 (right). OMV release is clearly observed in the right panel.

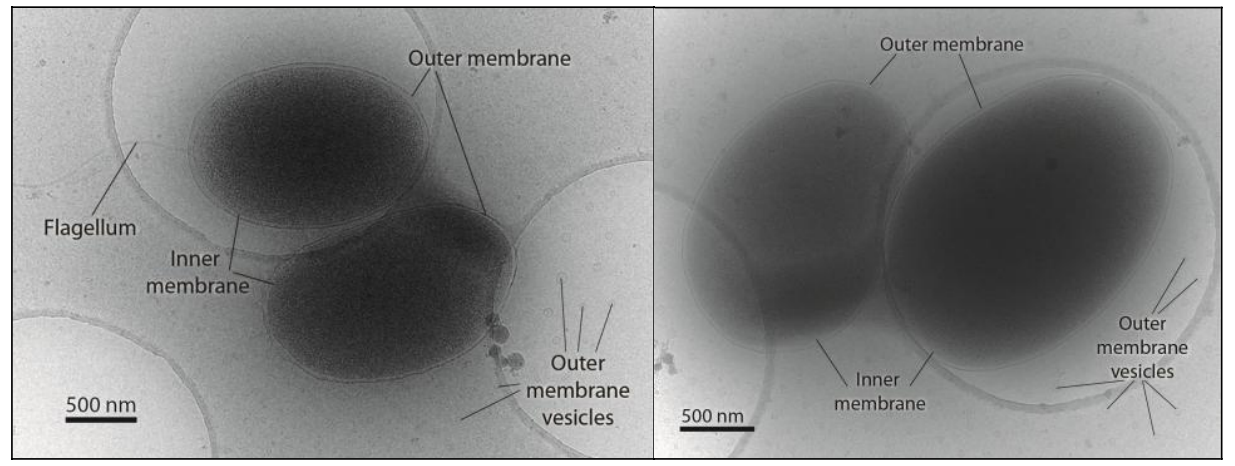

FIG. 4. V. vulnificus MO6-24/O (left) and CMCP6 (right). OMVs are observed forming concentric rings around the cells. 